\title{
Real-time observation of the intravalley spin-flip process in single-layer $\mathbf{W S}_{2}$
}

Zilong Wang ${ }^{1}$, Alejandro Molina-Sanchez ${ }^{2}$, Patrick Altmann ${ }^{1}$, Davide Sangalli ${ }^{3}$, Domenico De Fazio ${ }^{4}$, Giancarlo Soavi ${ }^{4}$, Ugo Sassi ${ }^{4}$, Federico Bottegoni ${ }^{1}$, Franco Ciccacci ${ }^{1}$, Marco Finazzi $^{1}$, Ludger Wirtz ${ }^{5}$, Andrea Ferrari ${ }^{4}$, Andrea Marini ${ }^{3}$, Giulio Cerullo ${ }^{1,6}$ and Stefano Dal Conte $^{1, *}$

\author{
${ }^{1}$ Dipartimento di Fisica, Politecnico di Milano, Piazza L. da Vinci 32, I-20133 Milano, Italy \\ ${ }^{2}$ Institute of Materials Science (ICMUV), University of Valencia, Catedràtico Beltràn 2, \\ E-46980, Valencia, Spain \\ ${ }^{3}$ Istituto di Struttura della Materia (ISM), CNR, Via Salaria Km 29.3, I-00016 \\ Monterotondo Stazione, Italy \\ ${ }^{4}$ Cambridge Graphene Centre, University of Cambridge, $9 \mathrm{JJ}$ Thomson Avenue, \\ Cambridge CB3 0FA, United Kingdom \\ ${ }^{5}$ Université du Luxembourg, 162 A, avenue de la Faïencerie, L-1511 Luxembourg \\ ${ }^{6}$ IFN-CNR, Piazza L. da Vinci 32, I-20133 Milano, Italy
}

\begin{abstract}
We use helicity-resolved transient absorption spectroscopy to track intravalley scattering dynamics in monolayer $\mathrm{WS}_{2}$. We find that spinpolarized carriers scatter from upper to lower conduction band by reversing their spin orientation on a sub-ps timescale.
\end{abstract}

In two-dimensional Transition Metal Dichalcogenides (TMDs) interactions with light are dominated by strongly bounded excitons due to the low-dimensional quantum confinement[1]. In Mo and $\mathrm{W}$ based TMDs, the large spin-orbit coupling lifts the spin degeneracy of the valence (VB) and the conduction bands (CB) giving rise to the A and $\mathrm{B}$ interband excitonic transitions[2]. Theory predicts the CB spin-ordering in Mo-based TMDs to be different from W-based TMDs [3]. In the former case, the spins of the electrons in the lowest $\mathrm{CB}$ and in the highest $\mathrm{VB}$ at $\mathrm{K} / \mathrm{K}^{\prime}$ are parallel, giving rise to an optically bright $\mathrm{A}$ exciton state while in the latter case they are antiparallel, resulting in a dark A exciton at a lower energy than the bright one (intravalley dark exciton, see left panel in Fig.1). The presence of dark excitons has been revealed indirectly from the observation of anomalous quenching of the PL emission at low temperature in single-layer (1L)- $\mathrm{WSe}_{2}$ [4].

Here we use two-colour helicity-resolved pump-probe spectroscopy to directly resolve the intravalley spin-flip process of the photoexcited electrons in the CB of $1 \mathrm{~L}-\mathrm{WS}_{2}$.

In our experiment, spin-polarized carriers are photo-injected by a circularly polarized pump resonant to the A exciton transition, while their temporal evolution is detected by a cocircularly polarized probe pulse resonant with either the A or B excitons (red and blue curves in the central panel in Fig. 1).

When the probe is resonant with the A exciton, we observe an instantaneous (i.e. limited by the temporal resolution $\sim 100 \mathrm{fs})$ build-up of the differential transmission $(\Delta \mathrm{T} / \mathrm{T})$ signal. This positive signal (photo-bleaching, PB) arise from Pauli-blocking in the A excitonic state. On the other hand, when the probe is resonant with the B exciton, the intravalley spinflip scattering process (grey arrow in left panel in Fig. 1) from upper to lower CB states causes a delayed formation (on a time scale $\tau_{\text {rise }}$ ) of the PB signal (blue curve in Fig.1b). We find that at $77 \mathrm{~K}$ this process occurs on a sub-ps time scale (i.e. $\sim 200 \mathrm{fs}$ ). 

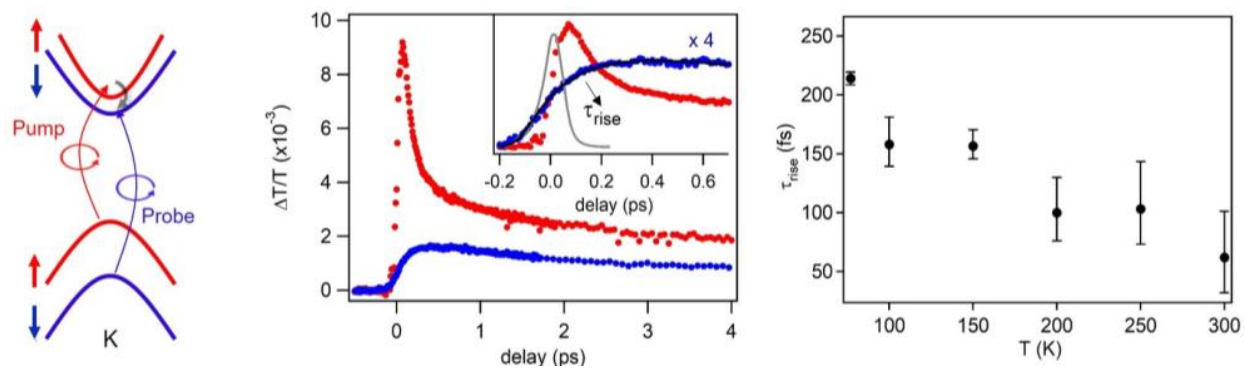

Fig. 1. (Left panel) Sketch of the band structure of $1 \mathrm{~L}-\mathrm{WS}_{2}$ around the $\mathrm{K}$ point of the Brillouin zone. The pump and probe pulses have the same circular polarization. The pump is resonant with the A exciton while the probe is resonant with either the A (degenerate configuration) or B exciton (nondegenerate configuration). Only the non-degenerate configuration is sketched for sake of clarity. Only excitons in $\mathrm{K}$ point are excited because of the valley dependent optical selection rules for circular polarization. The intravalley scattering process (grey arrow) from upper to lower CB state causes the reduction of the absorption of the probe beam by Pauli blocking and results in a delayed formation of the bleaching signal around the $\mathrm{B}$ exciton. (Central panel) The red and blue curves are the $\Delta \mathrm{T} / \mathrm{T}$ signal measured in $1 \mathrm{~L}-\mathrm{WS}_{2}$ at $77 \mathrm{~K}$ around the $\mathrm{A}$ and $\mathrm{B}$ excitonic transitions, following excitation of the A transition. For degenerate pump and probe energies, the build-up dynamics is pulse-width limited, while the non-degenerate configuration displays a delayed formation. In the inset, $\Delta \mathrm{T} / \mathrm{T}$ around the $\mathrm{B}$ exciton peak is multiplied by 4 to highlight the finite build up time. The grey line is the cross correlation function between the pump and probe pulses. (Right panel) Temperature dependence of the $\mathrm{B}$ exciton rise time $\tau_{\text {rise. }}$

Moreover, the strong dependence of $\tau_{\text {rise }}$ on the lattice temperature strongly suggests that this relaxation process is mediated by phonons (right panel of Fig. 1).

In order to get more insight into the origin and the dynamics of the measured intravalley relaxation process, we use first-principles calculations based on non-equilibrium manybody perturbation theory [5].

The band structure and the static absorption spectrum of $1 \mathrm{~L}-\mathrm{WS}_{2}$ are first calculated using density functional theory within local density approximation by taking into account the strong spin-orbit interaction (left panel in Fig. 2). The temporal dynamics is obtained by solving the Kadanoff-Baym equation for the one-body density matrix. In order to simulate our pump-probe experiments, we impose that the photoexcited carriers occupy only the upper $\mathrm{CB}$ state of the $\mathrm{K}$ valley at time zero, while at later times they can scatter into other electronic states by emitting phonons.

The right panel in Fig. 2 reports the temporal dynamics of the occupations of upper and lower CB states. This shows that the upper CB states are quickly depleted by efficient scattering processes mediated by phonons, while the lower ones are progressively filled on a temporal scale close to our experimental value.

In conclusion, our results shed light on the intravalley spin relaxation process in $1 \mathrm{~L}-\mathrm{WS}_{2}$, determining the formation of intravalley dark excitons on a sub-ps timescale. Dark excitons determine the light emission efficiency of $1 \mathrm{~L}-\mathrm{TMDs}$. For this reason, understanding the formation process of dark excitons is of valuable importance for designing 1L-TMDs based optoelectronic devices such light emitting diodes and polarized photon emitters. 

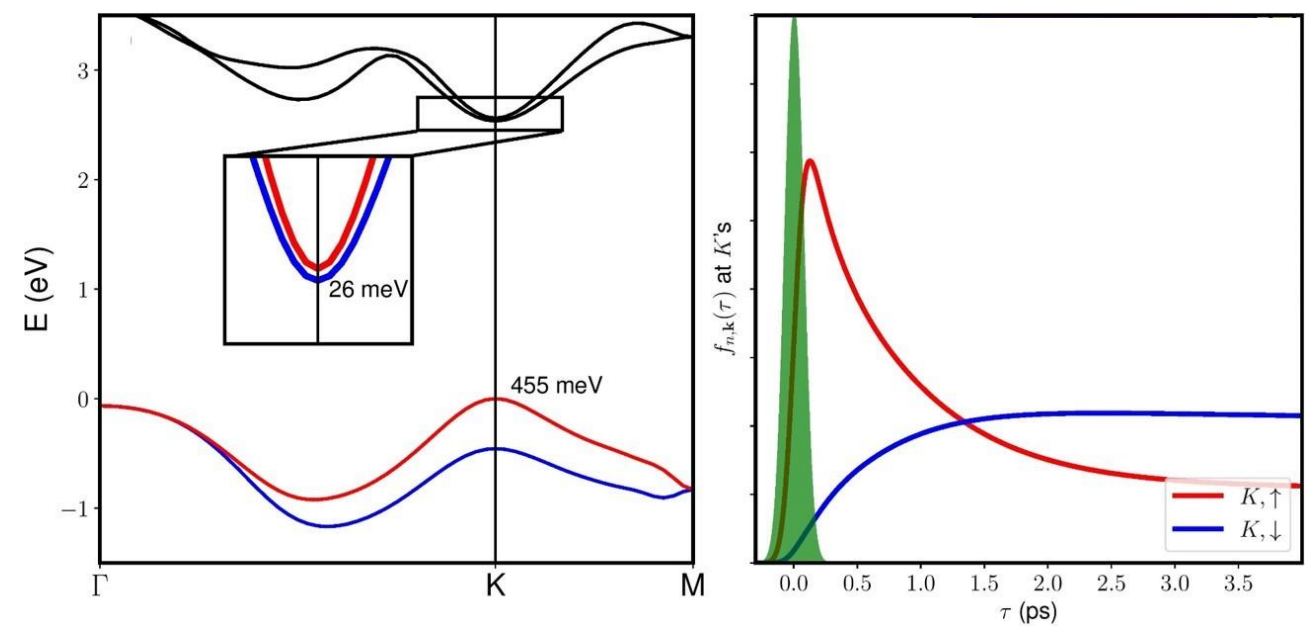

Fig. 2. (Left panel) Calculated band structure of $1 \mathrm{~L}-\mathrm{WS}_{2}$. The spin-orbit splitting of the valence band in the $\mathrm{K}$ point is in good agreement with the experimental value. In the inset the calculated splitting of the $\mathrm{CB}$ (i.e. $26 \mathrm{meV}$ ) is reported on an enlarged scale. (Right panel) Temporal evolution of the number of carriers in the upper and lower CB state (respectively $\mathrm{K}, \uparrow$ and $\mathrm{K}, \downarrow$ ) around the $\mathrm{K}$ point after excitation by a $100 \mathrm{fs}$ pump pulse (green curve) centered around the A exciton.

\section{References}

1. G. Wang, A. Chernikov, M. M. Glazov, T. F. Heinz, X. Marie, T. Amand, and B. Urbaszek, Rev. Mod. Phys. 90, 021001 (2018)

2. T. Cheiwchanchamnangij and W. R. L. Lambrecht, Phys. Rev. B 85, 205302 (2012)

3. K. Kosmider, J. W. Gonzalez and J. Fernandez-Rossier, Phys. Rev. B 88, 245436 (2013).

4. X.-X. Zhang, Y. You, S. Y. F. Zhao and T. F. Heinz, Phys. Rev. Lett. 115, 257403 (2015).

5. A. Molina-Sanchez, D. Sangalli, L. Wirtz and A. Marini, Nano Lett. 17, 4549 (2017) 